\title{
A Reverse Analytic Inequality for the Elementary Symmetric Function with Applications
}

\author{
Huan-Nan Shi ${ }^{1}$ and Jing Zhang ${ }^{2}$ \\ ${ }^{1}$ Department of Electronic Information, Teacher's College, Beijing Union University, Beijing 100011, China \\ ${ }^{2}$ Basic Courses Department, Beijing Union University, Beijing 100101, China
}

Correspondence should be addressed to Jing Zhang; zhangljing4@outlook.com

Received 31 October 2013; Accepted 17 December 2013

Academic Editor: Fernando Simões

Copyright (c) 2013 H.-N. Shi and J. Zhang. This is an open access article distributed under the Creative Commons Attribution License, which permits unrestricted use, distribution, and reproduction in any medium, provided the original work is properly cited.

We give a reverse inequality involving the elementary symmetric function by use of the Schur harmonic convexity theory. As applications, several new analytic inequalities for the $n$-dimensional simplex are established.

\section{Introduction}

Let $\mathbf{x}=\left(x_{1}, x_{2}, \ldots, x_{n}\right) \in \mathbb{R}^{n}=(-\infty,+\infty)^{n}$. The elementary symmetric functions are defined by

$$
\begin{array}{r}
E_{k}(\mathbf{x})=E_{k}\left(x_{1}, \ldots, x_{n}\right)=\sum_{1 \leq i_{1}<\cdots<i_{k} \leq n} \prod_{j=1}^{k} x_{i_{j}}, \\
k=1, \ldots, n,
\end{array}
$$

$E_{0}(\mathbf{x})=1$, and $E_{k}(\mathbf{x})=0$ for $k<0$ or $k>n$.

In 1997, Leng and Tang [1] in order to achieve higher dimensional generalization of the famous Pedoe inequality [2-5] presented the following analytic inequality.

Let $\mathbf{x} \in(0,1 / 2)^{n}$, and $E_{1}(\mathbf{x})=1$. Then

$$
E_{n-1}\left(\frac{1}{\mathbf{x}}-2\right)=E_{n-1}\left(\frac{1}{x_{1}}-2, \ldots, \frac{1}{x_{n}}-2\right) \geq n(n-2)^{n-1} .
$$

Chen et al. [6] gave an analytic proof which is skillful. By the method of successive adjustment, Fan [7] gave a simple elementary proof for inequality under conditions $\mathbf{x} \in(0,1)^{n}$, $n \geq 2$, and $E_{1}(\mathbf{x})=1$. Using the same method, Shi [8] extended inequality (2) to the cases of $E_{k}((1 / \mathbf{x})-2)$ for $k=$ $1,2, \ldots, n-1$, where $\mathbf{x} \in \mathbb{R}_{+}^{n}$.
Theorem A. Let $\mathbf{x} \in \mathbb{R}_{+}^{n}=(0,+\infty)^{n}, n \geq 2$ and $E_{1}(\mathbf{x})=1$. Then for $k=1,2, \ldots, n-1$, one has

$$
E_{k}\left(\frac{1}{\mathbf{x}}-2\right)=E_{k}\left(\frac{1}{x_{1}}-2, \ldots, \frac{1}{x_{n}}-2\right) \geq\left(\begin{array}{l}
n \\
k
\end{array}\right)(n-2)^{k},
$$

where $\left(\begin{array}{l}n \\ k\end{array}\right)=n ! / k !(n-k) !$

And Shi also pointed out that inequality (3) does not hold for $k=n$.

In 2002, using analytical methods, $\mathrm{Ma}$ and $\mathrm{Pu}$ [9] gave parameter extension of the inequality (3).

Theorem B. Let $\mathbf{x} \in \mathbb{R}_{+}^{n}, n, k \in \mathbb{N}=\{1,2, \ldots\}, \mu>0, n \geq 2$ and $E_{1}(\mathbf{x})=1$. Then for $n \geq k+\mu-1$, one has

$$
E_{k}\left(\frac{1}{\mathbf{x}}-\mu\right)=E_{k}\left(\frac{1}{x_{1}}-\mu, \ldots, \frac{1}{x_{n}}-\mu\right) \geq\left(\begin{array}{l}
n \\
k
\end{array}\right)(n-\mu)^{k} .
$$

In 2005, Li et al. [10] gave an elementary proof for Theorem $\mathrm{B}$ when $k \leq n-1$.

In this paper, by studying the Schur harmonic convexity of $E_{k}((1 / \mathbf{x})-\mu)$, we obtain a reverse of inequality (4) and accordingly establish the reverse of the corresponding simplex inequalities.

Our main result is the following theorem. 
Theorem 1. Let $\mathbf{x} \in \mathbb{R}_{+}^{n}, n, k \in \mathbb{N}, \mu>0, n \geq 2$. Then for $n \geq k+\mu-1, E_{k}((1 / \mathbf{x})-\mu)$ is Schur harmonically concave on $\Omega=\left\{\mathbf{x}: \mathbf{x} \in \mathbb{R}_{+}^{n}, E_{1}(\mathbf{x}) \leq 1\right\}$. When $E_{1}(\mathbf{x}) \leq 1$, one has

$$
E_{k}\left(\frac{1}{\mathbf{x}}-\mu\right)=E_{k}\left(\frac{1}{x_{1}}-\mu, \ldots, \frac{1}{x_{n}}-\mu\right) \leq\left(\begin{array}{l}
n \\
k
\end{array}\right)\left(\frac{1}{H}-\mu\right)^{k},
$$

where $H=n\left(\sum_{i=1}^{n} x_{i}^{-1}\right)^{-1}$ is the harmonic mean of $\mathbf{x}$.

Taking $\mu=2$ in Theorem 1, we infer the following corollary.

Corollary 2. Let $\mathbf{x} \in \mathbb{R}_{+}^{n}, n, k \in \mathbb{N}$, and $E_{1}(\mathbf{x}) \leq 1$. Then for $n \geq k+1$, one has

$$
E_{k}\left(\frac{1}{\mathbf{x}}-2\right)=E_{k}\left(\frac{1}{x_{1}}-2, \ldots, \frac{1}{x_{n}}-2\right) \leq\left(\begin{array}{l}
n \\
k
\end{array}\right)\left(\frac{1}{H}-2\right)^{k},
$$

where $H=n\left(\sum_{i=1}^{n} x_{i}^{-1}\right)^{-1}$ is the harmonic mean of $\mathbf{x}$.

Taking $k=n-1$ in Corollary 2, we obtain the following inequality.

Corollary 3. Let $\mathbf{x} \in \mathbb{R}_{+}^{n}, n \in \mathbb{N}$, and $E_{1}(\mathbf{x}) \leq 1$. Then for $n \geq 2$, one has

$$
\sum_{i=1}^{n} \prod_{j=1, j \neq i}^{n}\left(\frac{1}{x_{j}}-2\right) \leq n\left(\frac{1}{H}-2\right)^{n-1},
$$

where $H=n\left(\sum_{i=1}^{n} x_{i}^{-1}\right)^{-1}$ is the harmonic mean of $\mathbf{x}$.

\section{Definitions and Lemmas}

Definition 4 (see $[11,12])$. Let $\mathbf{x}=\left(x_{1}, \ldots, x_{n}\right)$ and $\mathbf{y}=\left(y_{1}\right.$, $\left.\ldots, y_{n}\right) \in \mathbb{R}^{n}$.

(i) $\mathbf{x}$ is said to be majorized by $\mathbf{y}$ (in symbols $\mathbf{x} \prec \mathbf{y}$ ) if $\sum_{i=1}^{k} x_{[i]} \leq \sum_{i=1}^{k} y_{[i]}$ for $k=1,2, \ldots, n-1$ and $\sum_{i=1}^{n} x_{i}=$ $\sum_{i=1}^{n} y_{i}$, where $x_{[1]} \geq \cdots \geq x_{[n]}$ and $y_{[1]} \geq \cdots \geq y_{[n]}$ are rearrangements of $\mathbf{x}$ and $\mathbf{y}$ in a descending order.

(ii) $\Omega \subseteq \mathbb{R}^{n}$ is called a convex set if $\left(\alpha x_{1}+\beta y_{1}, \ldots, \alpha x_{n}+\right.$ $\left.\beta y_{n}\right) \in \Omega$ for every $\mathbf{x}$ and $\mathbf{y} \in \Omega$, where $\alpha$ and $\beta \in[0,1]$ with $\alpha+\beta=1$.

(iii) Let $\Omega \subseteq \mathbb{R}^{n}$. The function $\varphi: \Omega \rightarrow \mathbb{R}$ is said to be a Schur convex function on $\Omega$ if $\mathbf{x} \prec \mathbf{y}$ on $\Omega$ implies $\varphi(\mathbf{x}) \leq \varphi(\mathbf{y}) . \varphi$ is said to be a Schur concave function on $\Omega$ if and only if $-\varphi$ is Schur convex.

Lemma 5 (see $[11,12])$. Let $\mathbf{x}=\left(x_{1}, x_{2}, \ldots, x_{n}\right) \in \mathbb{R}^{n}$. Then

$$
(A, \ldots, A) \prec\left(x_{1}, x_{2}, \ldots, x_{n}\right),
$$

where $A=(1 / n) \sum_{i=1}^{n} x_{i}$ is the arithmetic mean of $\mathbf{x}$.

Definition 6 (see $[11,12])$. Let $\Omega \subseteq \mathbb{R}^{n}$.

(i) $\Omega$ is called a symmetric set, if $\mathbf{x} \in \Omega$ implies $\mathbf{x} P \in \Omega$ for every $n \times n$ permutation matrix $P$. (ii) The function $\varphi: \Omega \rightarrow \mathbb{R}$ is called symmetric if for every permutation matrix $P, \varphi(\mathbf{x} P)=\varphi(\mathbf{x})$ for all $\mathbf{x} \epsilon$ $\Omega$.

Lemma 7 (see $\left[11\right.$, p. 84]). Let $\Omega \subset \mathbb{R}^{n}$ be symmetric and have a nonempty interior convex set $\Omega^{0}$, and let $\varphi: \Omega \rightarrow \mathbb{R}$ be continuous on $\Omega$ and differentiable in $\Omega^{0}$. Then $\varphi$ is the Schurconvex (Schur-concave) function if and only if $\varphi$ is symmetric on $\Omega$ and

$$
\left(x_{1}-x_{2}\right)\left(\frac{\partial \varphi(\mathbf{x})}{\partial x_{1}}-\frac{\partial \varphi(\mathbf{x})}{\partial x_{2}}\right) \geq 0(\leq 0)
$$

holds for any $\mathbf{x} \in \Omega^{0}$.

The Schur-convexity described the ordering of majorization; the order-preserving functions were first comprehensively studied by Issai Schur in 1923. It has important applications in analytic inequalities, combinatorial optimization, quantum physics, information theory, and so on. See [11].

In 2009, Chu and Lv [13] introduced the notion of harmonically Schur convex function and some interesting inequalities were obtained. The Schur harmonic convexity involving some special functions has been investigated; see, for example, [14-20].

Definition 8 (see [13]). Let $\Omega \subset \mathbb{R}_{+}^{n}$.

(i) A set $\Omega$ is said to be harmonically convex if $\mathbf{x y} /(\lambda \mathbf{x}+$ $(1-\lambda) \mathbf{y}) \in \Omega$ for every $\mathbf{x}, \mathbf{y} \in \Omega$ and $\lambda \in[0,1]$, where $\mathbf{x y}=\sum_{i=1}^{n} x_{i} y_{i}$ and $(1 / \mathbf{x})=\left(1 / x_{1}, \ldots, 1 / x_{n}\right)$.

(ii) A function $\varphi: \Omega \rightarrow \mathbb{R}_{+}$is said to be Schur harmonically convex on $\Omega$ if $1 / \mathbf{x} \prec 1 / \mathbf{y}$ implies $\varphi(\mathbf{x}) \leq$ $\varphi(\mathbf{y})$. A function $\varphi$ is said to be a Schur harmonically concave function on $\Omega$ if and only if $-\varphi$ is a Schur harmonically convex function.

Lemma 9 (see [13]). Let $\Omega \subset \mathbb{R}_{+}^{n}$ be a symmetric and harmonically convex set with inner points and let $\varphi: \Omega \rightarrow \mathbb{R}_{+}$ be a continuously symmetric function which is differentiable on $\Omega^{0}$. Then, $\varphi$ is Schur harmonically convex (Schur harmonically concave) on $\Omega$ if and only if

$$
\left(x_{1}-x_{2}\right)\left(x_{1}^{2} \frac{\partial \varphi(\mathbf{x})}{\partial x_{1}}-x_{2}^{2} \frac{\partial \varphi(\mathbf{x})}{\partial x_{2}}\right) \geq 0(\leq 0), \quad \mathbf{x} \in \Omega^{0} .
$$

Lemma 10. Let $\mathbf{x} \in \mathbb{R}_{+}^{n}, n, k \in \mathbb{N}, \mu>0, n \geq 2$, and $E_{1}(\mathbf{x}) \leq 1$. Then for $n \geq k+\mu-1$, one has

$$
E_{k}\left(\frac{1}{\mathbf{x}}-\mu\right) \geq 0 \text {. }
$$

Proof. Let $E_{1}(\mathbf{x})=\sigma \leq 1$. Then $E_{1}(\mathbf{x} / \sigma)=\left(x_{1} / \sigma\right)+\cdots+$ $\left(x_{n} / \sigma\right)=1$; and $n \geq k+\mu-1 \geq k+\sigma \mu-1$, from Theorem $\mathrm{B}$, we have

$$
\begin{aligned}
E_{k}\left(\frac{\sigma}{\mathbf{x}}-\sigma \mu\right) & =E_{k}\left(\frac{\sigma}{x_{1}}-\sigma \mu, \ldots, \frac{\sigma}{x_{n}}-\sigma \mu\right) \\
& \geq\left(\begin{array}{l}
n \\
k
\end{array}\right)(n-\sigma \mu)^{k},
\end{aligned}
$$


and then

$$
\begin{aligned}
E_{k}\left(\frac{1}{\mathbf{x}}-\mu\right) & =E_{k}\left(\frac{1}{x_{1}}-\mu, \ldots, \frac{1}{x_{n}}-\mu\right) \\
& \geq\left(\begin{array}{l}
n \\
k
\end{array}\right)\left(\frac{n-\sigma \mu}{\sigma}\right)^{k} \geq 0 .
\end{aligned}
$$

Lemma 10 is proved.

\section{Proof of Main Result}

Proof of Theorem 1. When $n \geq 2, k=1$, obviously, inequality (5) holds. When $n \geq 2, k \geq 2$, noting that

$$
\begin{aligned}
\varphi(\mathbf{x}):= & E_{k}\left(\frac{1}{\mathbf{x}}-\mu\right) \\
= & \left(\frac{1}{x_{1}}-\mu\right)\left(\frac{1}{x_{2}}-\mu\right) E_{k-2}\left(\frac{1}{x_{3}}-\mu, \ldots, \frac{1}{x_{n}}-\mu\right) \\
& +\left(\frac{1}{x_{1}}-\mu+\frac{1}{x_{2}}-\mu\right) E_{k-1}\left(\frac{1}{x_{3}}-\mu, \ldots, \frac{1}{x_{n}}-\mu\right) \\
& +E_{k}\left(\frac{1}{x_{3}}-\mu, \ldots, \frac{1}{x_{n}}-\mu\right)
\end{aligned}
$$

we have

$$
\begin{aligned}
\frac{\partial \varphi(\mathbf{x})}{\partial x_{1}}= & -\frac{1}{x_{1}^{2}}\left(\frac{1}{x_{2}}-\mu\right) E_{k-2}\left(\frac{1}{x_{3}}-\mu, \ldots, \frac{1}{x_{n}}-\mu\right) \\
& -\frac{1}{x_{1}^{2}} E_{k-1}\left(\frac{1}{x_{3}}-\mu, \ldots, \frac{1}{x_{n}}-\mu\right), \\
\frac{\partial \varphi(\mathbf{x})}{\partial x_{2}}= & -\frac{1}{x_{2}^{2}}\left(\frac{1}{x_{1}}-\mu\right) E_{k-2}\left(\frac{1}{x_{3}}-\mu, \ldots, \frac{1}{x_{n}}-\mu\right) \\
& -\frac{1}{x_{2}^{2}} E_{k-1}\left(\frac{1}{x_{3}}-\mu, \ldots, \frac{1}{x_{n}}-\mu\right) .
\end{aligned}
$$

And then

$$
\begin{aligned}
\Delta & :=\left(x_{1}-x_{2}\right)\left(x_{1}^{2} \frac{\partial \varphi(\mathbf{x})}{\partial x_{1}}-x_{2}^{2} \frac{\partial \varphi(\mathbf{x})}{\partial x_{2}}\right) \\
& =-\frac{\left(x_{1}-x_{2}\right)^{2}}{x_{1} x_{2}} E_{k-2}\left(\frac{1}{x_{3}}-\mu, \ldots, \frac{1}{x_{n}}-\mu\right) .
\end{aligned}
$$

Now we distinguish two cases to prove $\Delta \leq 0$.

Case 1. If $n \geq 2, k=2$, then from the definition of elementary symmetric functions, it follows that $\Delta \leq 0$.

If $n=3, k=3$, then from the condition $n \geq k+\mu-1$, it follows that $\mu \leq n-k+1=1$, and by $x_{i} \leq E_{1}(\mathbf{x}) \leq 1$, it follows that $1 / x_{i} \geq 1, i=3, \ldots, n$; therefore,

$$
\begin{aligned}
E_{k-2} & \left(\frac{1}{x_{3}}-\mu, \ldots, \frac{1}{x_{n}}-\mu\right) \\
& =E_{1}\left(\frac{1}{x_{3}}-\mu, \ldots, \frac{1}{x_{n}}-\mu\right) \\
& =\sum_{i=3}^{n} \frac{1}{x_{i}}-(n-2) \mu \geq 0 .
\end{aligned}
$$

Case 2. If $n \geq 4$; then $n-2 \geq 2$, from $n \geq k+\mu-1$, it follows that

$$
(n-2) \geq(k-2)+\mu-1
$$

and $\sum_{i=3}^{n} x_{i} \leq 1$. So by Lemma 10 , it follows that $E_{k-2}\left(\left(1 / x_{3}\right)-\right.$ $\left.\mu, \ldots,\left(1 / x_{n}\right)-\mu\right) \geq 0$.

Thus, for all $n \geq 2, \Delta \leq 0$. By Lemma 9 , we can derive that $E_{k}((1 / \mathbf{x})-\mu)$ is Schur harmonically concave on $\Omega$.

From Lemma 5 , it is seen that

$$
\left(\frac{1}{H}, \ldots, \frac{1}{H}\right) \prec\left(\frac{1}{x_{1}}, \ldots, \frac{1}{x_{n}}\right) .
$$

According to Definition 8(ii), inequality (5) follows.

The proof of Theorem 1 is completed.

\section{Applications in Geometry}

Let $\Omega_{n}$ be an $n$-dimensional simplex in the $n$-dimensional Euclidean space $\mathbb{R}^{n}(n \geq 2)$ with vertices $A_{0}, A_{1}, \ldots, A_{n}$ whose volume is $V$. For $i=0,1, \ldots, n$, let $r_{i}$ be the radius of $i$ th escribed sphere of $\Omega_{n}$, and $F_{i}$ the area of the $i$ th face $f_{i}=\left\{A_{0}, A_{1}, \ldots, A_{i-1}, A_{i+1}, \ldots, A_{n}\right\}$ of $\Omega_{n}, F=\sum_{i=0}^{n} F_{i} . R$ and $r$ are circumradius and inradius of $\Omega_{n}$, respectively. Let $P$ be an arbitrary interior point of the simplex $\Omega_{n}, d_{i}$ the distance from the point $P$ to the $i$ th face $f_{i}$ of $\Omega_{n}$, and $h_{i}$ the altitude of $\Omega_{n}$ from vertex $A_{i}$ for $i=0,1, \ldots, n$.

In [9], by Theorem $\mathrm{B}, \mathrm{Ma}$ and $\mathrm{Pu}$ obtained the following two theorems.

Theorem C. In the simplex $\Omega_{n}(n \geq 2)$, for $k=1,2, \ldots, n$, one has

$$
E_{k}\left(\frac{h_{0}}{r_{0}}, \frac{h_{1}}{r_{1}}, \ldots, \frac{h_{n}}{r_{n}}\right) \geq\left(\begin{array}{c}
n+1 \\
k
\end{array}\right)(n-1)^{k}
$$

with equality if and only if $\Omega_{n}$ is an orthocentric simplex.

Theorem D. Let $P$ be an arbitrary point in the interior of $n$ dimensional simplex $\Omega_{n}$. Let $B_{i}$ be the intersection of the line $A_{i} P$ with the hyperplane $f_{i}$; further let $R_{i}=\left|A_{i} P\right|, d_{i}^{\prime}=\left|P B_{i}\right|$, $M_{i}=\left|A_{i} B_{i}\right|, i=0,1, \ldots, n$. Then for $k=1,2, \ldots, n+1$, one has

$$
E_{k}\left(\frac{R_{0}}{d_{0}^{\prime}}, \frac{R_{1}}{d_{1}^{\prime}}, \ldots, \frac{R_{n}}{d_{n}^{\prime}}\right) \geq\left(\begin{array}{c}
n+1 \\
k
\end{array}\right) n^{k}
$$

with equality if and only if $\Omega_{n}$ is an orthocentric simplex, and $P$ is its orthocentre.

Now, by Theorem 1, we give the reverse of inequalities (21) and (22).

Theorem 11. In the simplex $\Omega_{n}$, for $k=1,2, \ldots, n$, one has

$$
E_{k}\left(\frac{h_{0}}{r_{0}}, \frac{h_{1}}{r_{1}}, \ldots, \frac{h_{n}}{r_{n}}\right) \leq\left(\begin{array}{c}
n+1 \\
k
\end{array}\right)\left(\frac{1}{H}-2\right)^{k} \text {, }
$$

where $H=(n+1)\left(\sum_{i=0}^{n}\left(\left(h_{i} / r_{i}\right)+2\right)\right)^{-1}$. 
Proof. In the $n$-dimensional simplex, for $k=0,1, \ldots, n$, it is well known that

$$
\begin{gathered}
r_{i}=\frac{n V}{\sum_{j=0}^{n} F_{j}-2 F_{i}}, \\
h_{i}=\frac{n V}{F_{i}} .
\end{gathered}
$$

Let $x_{i}=F_{i} / \sum_{j=0}^{n} F_{j}, i=0,1, \ldots, n$. Then, $E_{1}(\mathbf{x})=1$. From equalities (24), we have

$$
\frac{1}{x_{i}}-2=\frac{\sum_{j=0}^{n} F_{j}-2 F_{i}}{F_{i}}=\frac{h_{i}}{r_{i}}, \quad i=0,1, \ldots, n .
$$

Thus from the inequality (6), it is deduced that the inequality (23) holds.

The proof of Theorem 11 is completed.

Theorem 12. Let $P$ be an arbitrary point in the interior of $n$ dimensional simplex $\Omega_{n}$. Let $B_{i}$ be the intersection of the line $A_{i} P$ with the hyperplane $f_{i}$; further let $R_{i}=\left|A_{i} P\right|, d_{i}^{\prime}=\left|P B_{i}\right|$, $M_{i}=\left|A_{i} B_{i}\right|, i=0,1, \ldots, n$. Then, for $k=1,2, \ldots, n+1$,

$$
E_{k}\left(\frac{R_{0}}{d_{0}^{\prime}}, \frac{R_{1}}{d_{1}^{\prime}}, \ldots, \frac{R_{n}}{d_{n}^{\prime}}\right) \leq\left(\begin{array}{c}
n+1 \\
k
\end{array}\right)\left(\frac{1}{H}-1\right)^{k},
$$

where $H=(n+1)\left(\sum_{i=0}^{n}\left(M_{i} / d_{i}^{\prime}\right)\right)^{-1}$.

Proof. It is easy to see that

$$
\sum_{i=0}^{n} \frac{d_{i}^{\prime}}{M_{i}}=1, \quad \frac{R_{i}}{M_{i}}=1-\frac{d_{i}^{\prime}}{M_{i}}, \quad i=0,1, \ldots, n
$$

In the inequality (5), let $\mu=1, x_{i}=d_{i}^{\prime} / M_{i}$. Then, $1 \leq k \leq$ $n+1,\left(1 / x_{i}\right)-1=R_{i} / d_{i}^{\prime}, i=0,1, \ldots, n$. Thus, inequality (26) holds.

The proof of Theorem 12 is completed. ing.

When $k=n+1$, inequality (26) is reduced to the follow-

Corollary 13. Under the conditions of Theorem 12, one has

$$
\prod_{i=0}^{n} R_{i} \leq\left(\frac{1}{H}-1\right)^{n+1} \prod_{i=0}^{n} d_{i}^{\prime} .
$$

Theorem 14. In the simplex $\Omega_{n}$, let

$$
\begin{array}{r}
\lambda_{i}=\frac{F-2 F_{i}}{F_{i}}, \quad \mu_{i}=\frac{F_{0}^{2}+\cdots+F_{n}^{2}-2 F_{i}^{2}}{F_{i}^{2}}, \\
(i=0,1, \ldots, n) .
\end{array}
$$

Then,

$$
\sum_{i=0}^{n} \prod_{j=0, j \neq i}^{n} \lambda_{j} \leq(n+1)\left(\frac{1}{H}-2\right)^{n}
$$

where $H=n\left(\sum_{i=0}^{n}\left(\lambda_{i}+2\right)\right)^{-1}$;

$$
\sum_{i=0}^{n} \prod_{j=0, j \neq i}^{n} \mu_{j} \leq(n+1)\left(\frac{1}{H}-2\right)^{n},
$$

where $H=n\left(\sum_{i=0}^{n}\left(\mu_{i}+2\right)\right)^{-1}$.

Proof. Taking $x_{i}=\left(F_{i} / F\right)(i=0,1, \ldots, n)$ and applying Corollary 3 for these $n+1$ positive real numbers $x_{0}, x_{1}, \ldots, x_{n}$, inequality (30) follows.

Taking $x_{i}=F_{i}^{2} /\left(F_{0}^{2}+F_{1}^{2}+\cdots+F_{n}^{2}\right)$ and applying Corollary 3 , the desired inequality (31) follows.

The proof of Theorem 14 is completed.

Remark 15. Inequalities (30) and (31) give the reverse of the inequalities (2.10) and (2.11) in [10], respectively.

\section{Conflict of Interests}

The authors declare that there is no conflict of interests regarding the publication of this paper.

\section{Acknowledgments}

The work was supported by the Importation and Development of High-Caliber Talents Project of Beijing Municipal Institutions (IDHT201304089) and the Funding Project for Academic Human Resources Development in Institutions of Higher Learning under the Jurisdiction of Beijing Municipality (PHR (IHLB)) (PHR201108407). The authors are grateful to Professor Fernando Simões and the four anonymous reviewers for helpful pieces of advice that improved its presentation.

\section{References}

[1] G. S. Leng and L. H. Tang, "Some generalizations to several dimensional of the Pedoe inequality with applications," Acta Mathematica Sinica, vol. 40, no. 1, pp. 14-21, 1997 (Chinese).

[2] L. Yang and J. Z. Zhang, "Generalization to higher dimensions of the Neuberg-Pedoe inequqlity with applitions," Acta Mathematica Sinica, vol. 24, no. 3, pp. 401-408, 1981 (Chinese).

[3] L. Yang and J. Z. Zhang, "A generalisation to several dimensions of the Neuberg-Pedoe inequality, with applications," Bulletin of the Australian Mathematical Society, vol. 27, no. 2, pp. 203-214, 1983.

[4] D. S. Mitrinović and J. E. Pečarić, "About the Neuberg-Pedoe and the Oppenheim inequalities," Journal of Mathematical Analysis and Applications, vol. 129, no. 1, pp. 196-210, 1988.

[5] D. S. Mitrinović, J. E. Pečarić, and V. Volenec, Recent Advances in Geometric Inequalities, Kluwer Academic, Dordrecht, The Netherlands, 1989.

[6] J. Chen, Y. Huang, and S. H. Xia, "A note on generalizations of Neuberg-Pedoe inequality to higher dimensional case," Journal of Sichuan University, vol. 36, no. 2, pp. 197-200, 1999 (Chinese).

[7] Y. W. Fan, "An generalization of Neuberg-Pedoe inequality for simplies," Journal of Yanan University, vol. 18, no. 3, pp. 19-22, 1999 (Chinese).

[8] H. N. Shi, "The generalization of a analytic inequality," Journal of Chengdu University, vol. 20, no. 1, pp. 5-7, 2001 (Chinese). 
[9] T. Y. Ma and Z. N. Pu, "Some generalizations of an analytical inequality and their applications," Journal of Sichuan University, vol. 39, no. 1, pp. 1-6, 2002 (Chinese).

[10] X. Y. Li, G. S. Leng, and L. H. Tang, "Inequalities for a simplex and any point," Mathematical Inequalities \& Applications, vol. 8, no. 3, pp. 547-557, 2005.

[11] A. W. Marshall, I. Olkin, and B. C. Arnold, Inequalities: Theory of Majorization and Its Application, Springer, New York, NY, USA, 2nd edition, 2011.

[12] B. Y. Wang, Foundations of Majorization Inequalities, Beijing Normal University, Beijing, China, 1990, (Chinese).

[13] Y. M. Chu and Y. P. Lv, "The Schur harmonic convexity of the Hamy symmetric function and its applications," Journal of Inequalities and Applications, vol. 2009, Article ID 838529, 10 pages, 2009.

[14] W. F. Xia and Y. M. Chu, "The Schur harmonic convexity of Lehmer means," International Mathematical Forum, vol. 4, no. 41-44, pp. 2009-2015, 2009.

[15] Y. M. Chu and T. C. Sun, "The Schur harmonic convexity for a class of symmetric functions," Acta Mathematica Scientia B, vol. 30, no. 5, pp. 1501-1506, 2010.

[16] Y.-M. Chu and W.-F. Xia, "Necessary and sufficient conditions for the Schur harmonic convexity of the generalized Muirhead mean," Proceedings of A. Razmadze Mathematical Institute, vol. 152, pp. 19-27, 2010.

[17] Z.-H. Yang, "Schur harmonic convexity of Gini means," International Mathematical Forum, vol. 6, no. 13-16, pp. 747-762, 2011.

[18] W.-F. Xia, Y.-M. Chu, and G.-D. Wang, "Necessary and sufficient conditions for the Schur harmonic convexity or concavity of the extended mean values," Revista de la Unión Matemática Argentina, vol. 51, no. 2, pp. 121-132, 2010.

[19] H. N. Shi, Theory of Majorization and Analytic Inequalities, Harbin Institute of Technology, Harbin, China, 2012, (Chinese).

[20] H. N. Shi and J. Zhang, "Some new judgement theorems of Schur geometric and Schur harmonic convexities for a class of symmetric functions," Journal of Inequalities and Applications, vol. 2013, article 527, 2013. 


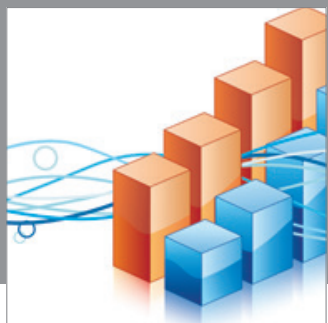

Advances in

Operations Research

mansans

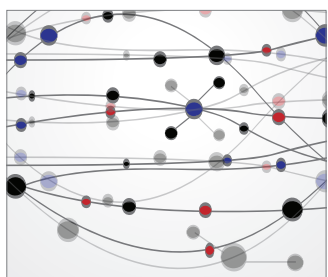

The Scientific World Journal
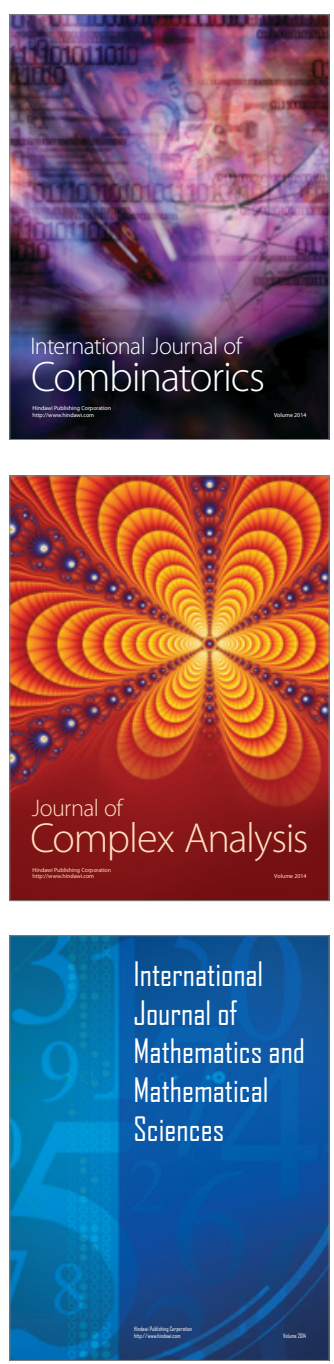
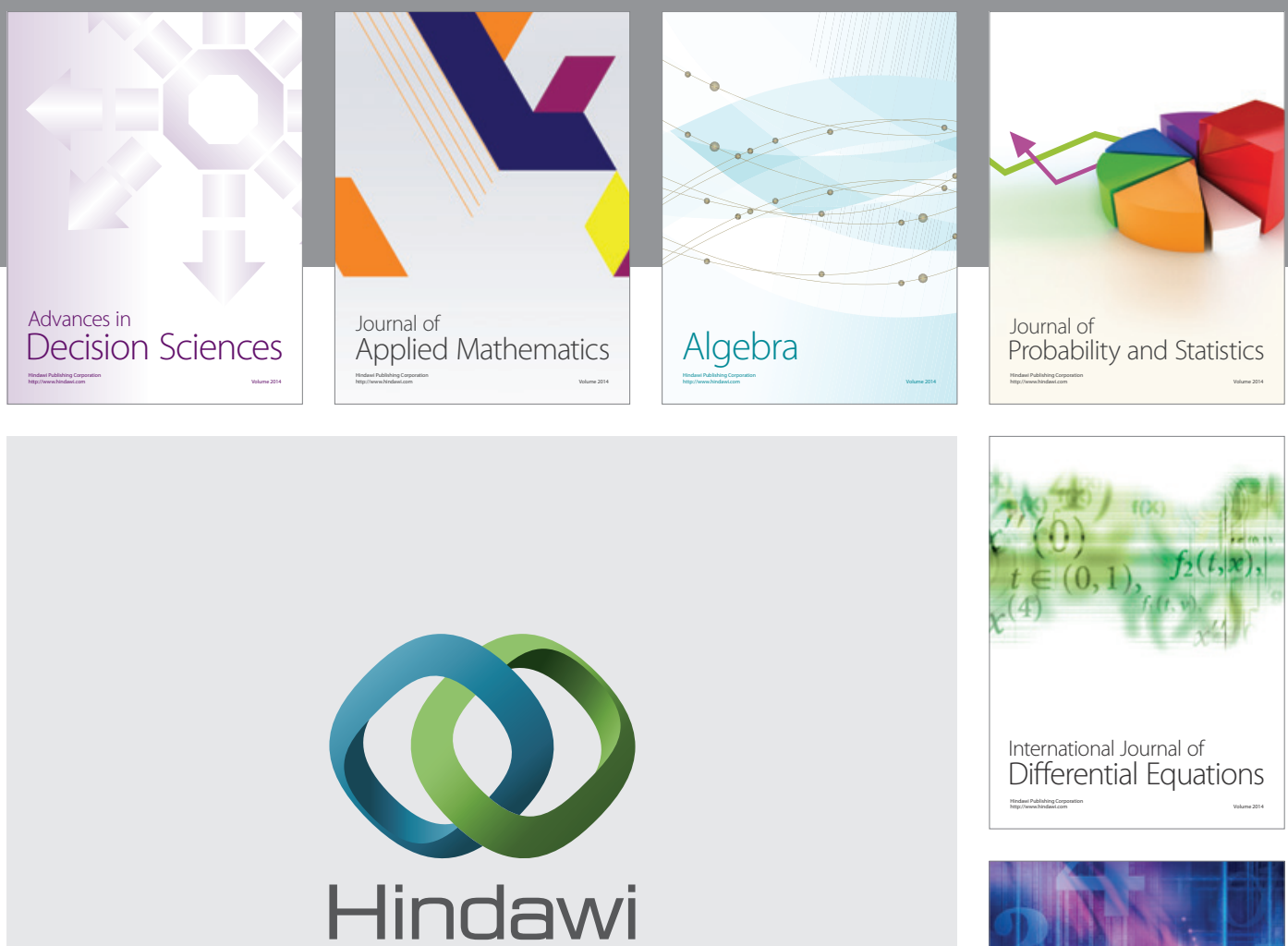

Submit your manuscripts at http://www.hindawi.com
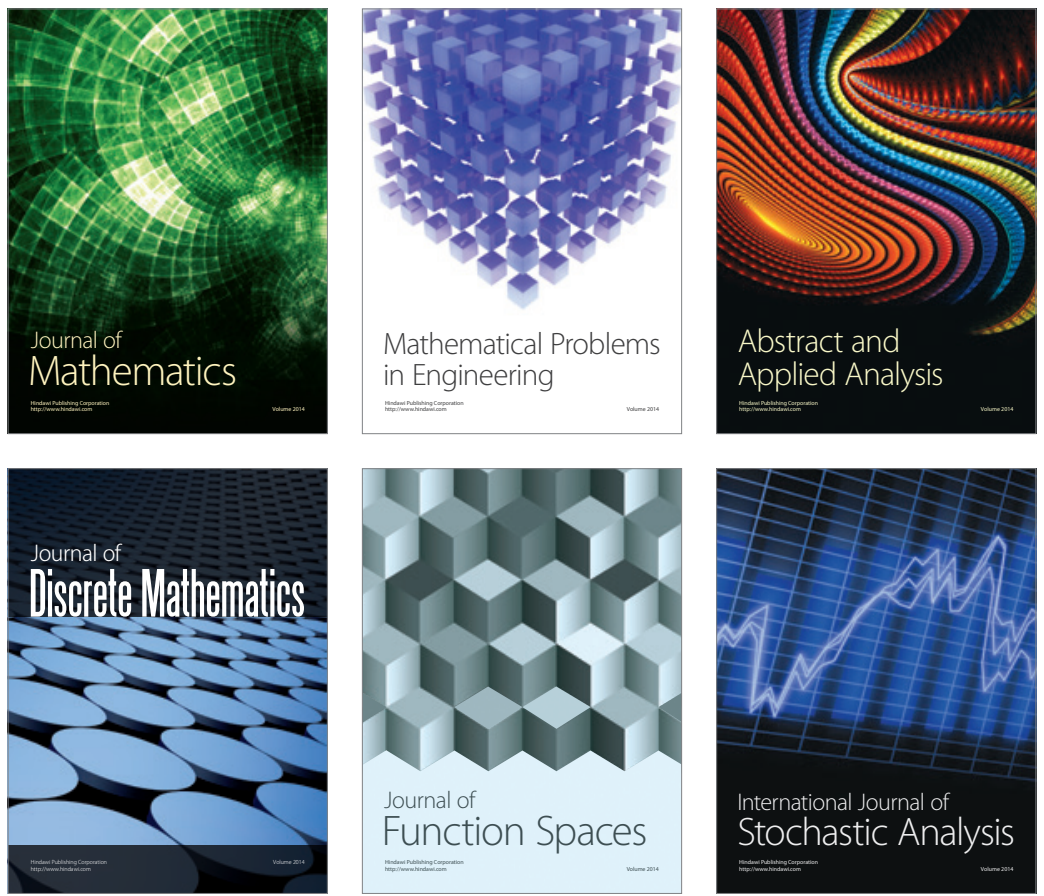

Journal of

Function Spaces

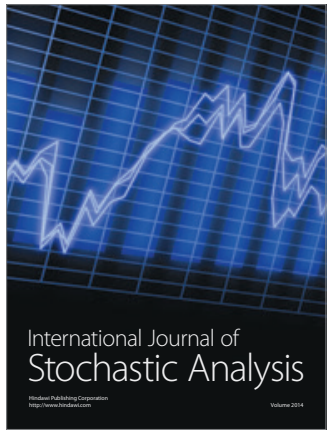

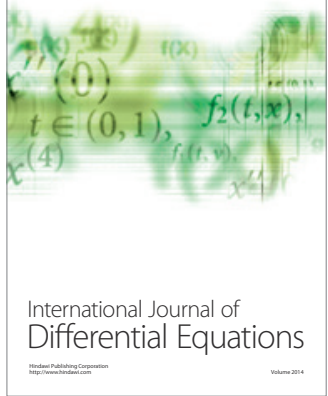
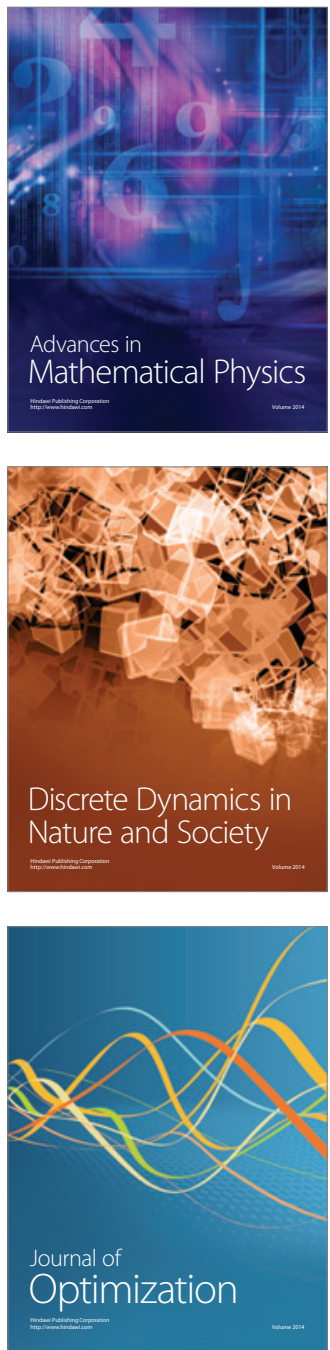